\title{
INVESTIGATION OF DATA MINING METHOD FOR THERMAL-FLUID SIMU- LATION RESULTS BASED ON PRESSURE DIFFERENCE BETWEEN FRONT AND BACK SIDES USING MEDIAL-SURFACE AND ITS APPLICATION TO THERMAL DESIGN PROBLEMS
}

\author{
N. Isoshima ${ }^{1}$, M. Onodera ${ }^{1}$, and S. Obayashi ${ }^{2}$ \\ ${ }^{1}$ Hitachi Research Laboratory, Hitachi. Ltd. (nobuyuki.isoshima.em@ hitachi.com) \\ ${ }^{2}$ Institute of Fluid Science, Tohoku University
}

\begin{abstract}
A data mining method is proposed for extracting feature regions and for suggesting design candidates from large-scale Computational Fluid Dynamics (CFD) results. The detected regions in this method are the regions where the pressure difference between the front and back sides is large. We introduce a medial-surface used in the structural analysis for the thin parts as an evaluation surface in order to apply complicated geometries, which have curved surfaces, holes, ribs, and steps. We applied this method to the laser diode (LD) cooling problem in optical disc drives, and the following results were obtained. (1) Three featureregions are extracted from the CFD results. (2) Two of three regions were effective and were consistent with the actual product design.
\end{abstract}

Keywords: Data mining, Medial-surface, Pressure difference, Thermal design.

\section{INTRODUCTION}

Many factors such as high-performance, low cost, and short development period are demanded when designing a product. The effective use of digital engineering tools such as CAD and CAE is important. For electronic and IT equipments such as optical disc drives, blade servers, flat panel TVs, and Liquid Crystal Display (LCD) projectors, the main market needs are downsizing, high-speed, and high-density processing/recording, and high-brightness. This means that the heat-generating density within the body of a given product is increasing. On the other hand, the product cycle is shortening. Therefore, process and product innovation for a more effective thermal design is required. Furthermore, it is difficult to stably generate boundary-fitting structured or unstructured grids for thermal-fluid simulation that are high quality in a short amount of time, because of their geometric complexity.

Hitachi has been developing a simulation system based on the Cartesian grid method and applying it to various product developments to solve these problems [1]-[2]. In those cases, the entire body of the product is divided into 107- 108 order grids using the Cartesian grid. The important thing in such developments lies in (1) finding some useful knowledge from the 
simulation results data, and (2) quick proper reflection to the product structure. However, the complexity of the internal structure creates highly complicated flow pattern for electronic and IT equipment. So, to beat the geometric and physical complexity of large amount of simulation data, a new problem has arisen which is to build a new approach to assist in the engineer's understanding of the simulation results and their creativity.

The purpose of this study is to build a data mining system that extracts the feature regions from large amounts of data and proposes alternative structural modifications with an expected-effectiveness ranking. The authors expect this system to help salvage effective knowledge and create new design ideas from overlooked data. In this paper, a new data mining system based on the empirical design knowledge is discussed, and the results of the extraction of the feature regions and the verification of the proposed design candidates are reported.

\section{DATA MINING METHOD THAT USES EMPIRICAL COOLING DESIGN KNOWLEDGE}

There are two major empirical principles in the thermal design. They are as follows.

(1) Specify the regions where the thermal resistance is large and reduce it.

(2) Specify the regions where the ventilation resistance is large and reduce it [3].

Other methods often used in thermal design adjust the position and size of the intake and exhaust port housing [4], or add an opening hole around the heat generating parts to improve the local ventilation. These are often carried out in experimental studies using a protomodel. When considering this process in hydrodynamics, the search process is conducted where the pressure difference between the front and back sides is large and the new flow near the heat generating parts would be induced by adding new openings. If we examine this process using CFD simulations, the data mining process results above process numerically.

However, there are some issues. The most difficult issue is how to evaluate the pressure difference between the front and back sides for arbitrarily shaped structures. Electronic and IT equipment are composed of many sheet parts made of resin or metal, which include many irregularities, curved surfaces, small ribs, and the holes for reinforcement. This makes it difficult to determine the evaluation surfaces for determining the pressure difference, and thus, an established method for determining all this has yet to be established. A novel approach is proposed in this study that can set the evaluation surface for complicated geometries and uses the data mining process to find the feature regions where the pressure difference is large and the opening hole is effective enough for cooling.

We adopted a medial-surface in this study to evaluate the pressure difference between the front and back sides of arbitrarily complicated geometries. The medial-surface is a virtual surface with a zero thickness, which locates on the mid-surface between the front and back sides, or the front side, or back side. The medial-surface is widely used in structural and vibration analysis to maintain the analysis precision of thin-thickness parts, but it is not used for CFD simulation. Onodera et al. developed a medial-surface generation system for not only 
parts but also assembly level models [5]. Figure 1shows examples of a solid and medialsurface model that are generated for structure analysis. Appropriate medial-surfaces are generated for complicated geometries with holes and steps.

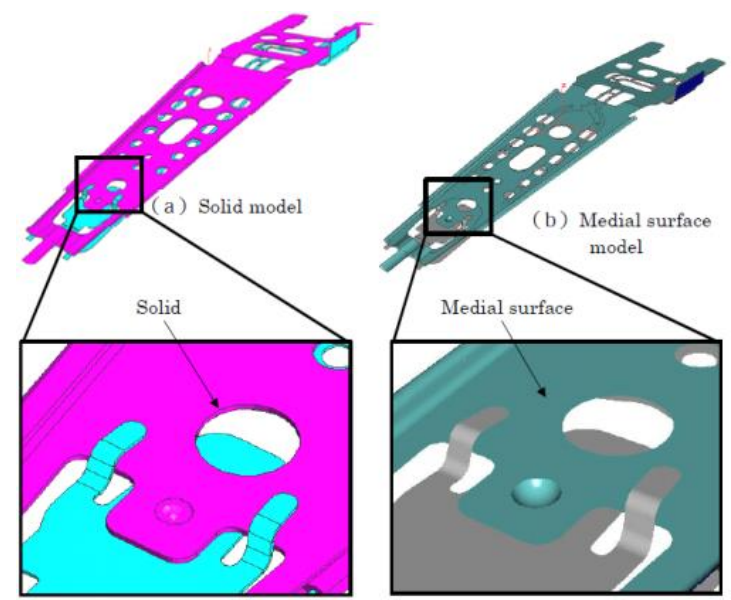

Figure 1. Sample of medial-surface model for HDD head parts

A concrete method is explained using Figure 2. First, a 3D CFD simulation is executed using a 3D model with the thickness. Secondly, the medial-surface model is generated from the 3D model, and the medial-surface model is divided into elements. Next, the normal projection points from the medial-surface to the front/back sides are calculated for each other, and the represent surface values are linearly incorporated by the adjacent surface-element values. Then, the values are projected to the medial-surface's elements as the pressure values of the front/back sides on the 3D model, and the pressure difference value is calculated for the medial-surface elements. In our method, the medial-surface elements store both the front and back side pressure values, and furthermore the pressure difference values.

After the pressure difference distribution is obtained, the data mining process evaluates the contribution to the convective cooling effect from the back table. One method that is highly reliable incorporates additional CFD simulations that are executed for the added opening-hole models. However, this takes too much time. In this study, in order to avoid a longer simulation time, the following two simple evaluation indexes are adopted.

(1) The distance from the specified heat generating part to the opening-hole candidates: higher cooling effect would be gained in short distance order.

(2) The integral area value of the pressure difference for opening-hole candidates: higher cooling effect would be gained in large integral area value order. 


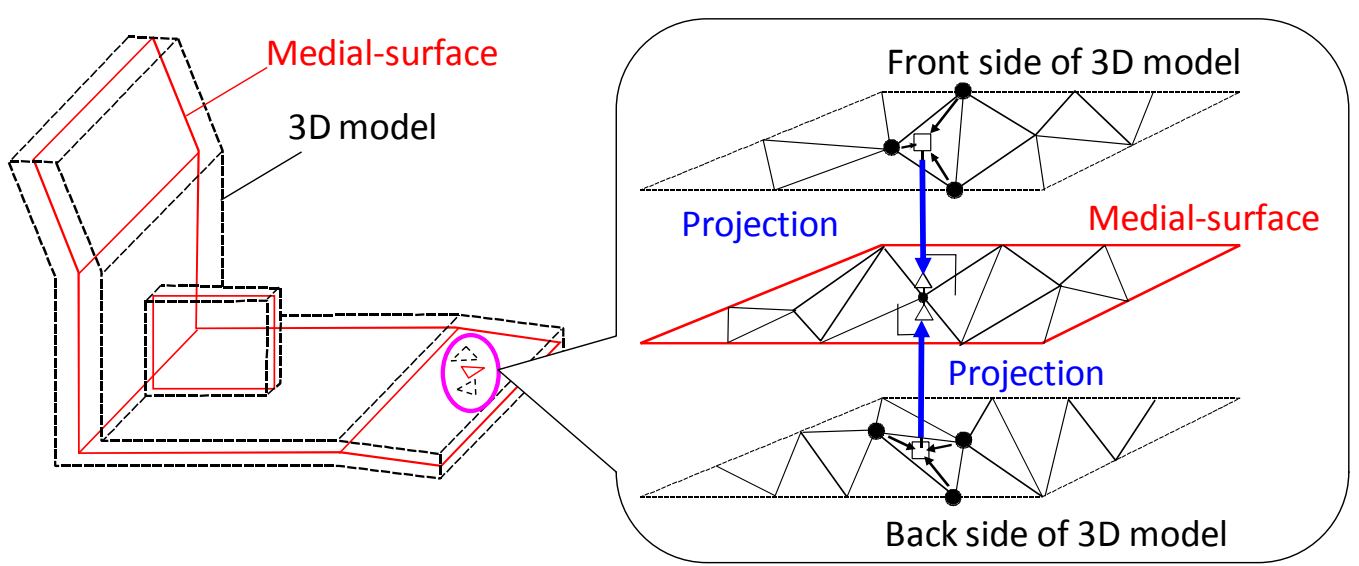

(a) Projection pressure values from surfaces of 3D model to medial-surface

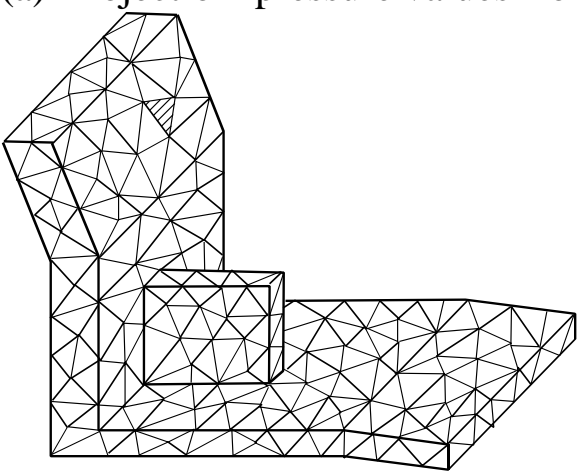

(b) 3D model

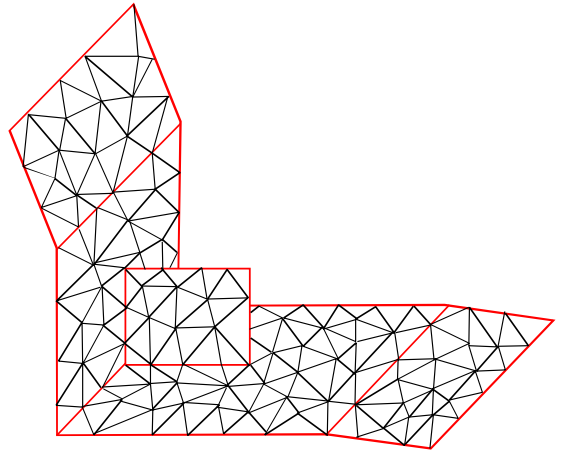

(c) Medial-surface model

Figure 2. Schematic of pressure value projection

\section{VERIFICATION OF COOLING DESIGN FOR OPTICAL DISC DRIVE}

The proposed data mining method is applied for the cooling design of an optical disc drive previously developed by one of the authors, and the results verify whether the reasonable candidates are chosen or not. Here, the CFD simulation for the cooling design is done using a Cartesian grid that is equally spaced $0.3 \mathrm{~mm}$ high, $0.5 \mathrm{~mm}$ in the other direction and has a total of about 30 million elements. In the flow solver, the continuity equation and NavierStokes equation for an unsteady incompressible flow are solved by using HSMAC method using a standard Large Eddy Simulation (LES). The simulation results were already validated by conducting experiments to take pressure and velocity distribution measurements using Particle Image Velocimetry (PIV). Although there were some errors due to the Cartesian grid, we confirmed that reasonable simulation results can be obtained when investigating the thermal design [6].

In the optical disc drives shown in Figure 3, a laser light is irradiated from the LD inside the optical pick-up under the disc to the high-speed $(9,000 \mathrm{~min}-1)$ rotating disc that is 120 $\mathrm{mm}$ in diameter, then the data is read or recorded. The main thermal design point is the heat dissipation from the LD and the lifetime warranty period against temperature increases of the LD. 


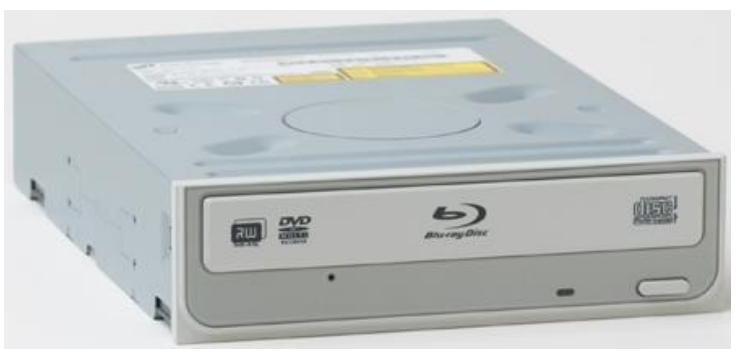

(a) Outline view

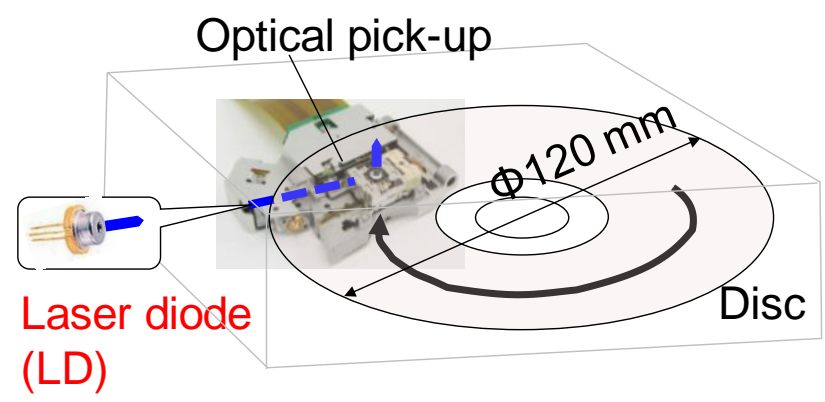

(b) Internal schematic view

Figure 3. Optical disc drive

The Blu-ray Disc (BD) drive that achieved BD 4X-speed recording and DVD-R 12X speed recording for the first time in the industry in July 2006 was selected to verify the validity of the data mining method. One of the authors developed a new LD cooling structure using CFD simulations [1]. From the simulation results, the author found that the low pressure region induced by the disk rotation is locally formed on the tray, where it is just above the position of the LD. Then, the additional hole on the tray is opened, and a secondary upward flow is induced by the pressure difference between the upper and lower sides of the tray and this promotes the cooling ventilation of the LD. The new structure achieves an approximately $2 \mathrm{~K}$ reduction of the temperature rise of the LD without needing any additional components such as a fan. This $2 \mathrm{~K}$ temperature reduction for a LD is significant, considering that the LDtemperature rise is often designed to be under $10-15 \mathrm{~K}$ compared to the reference air temperature in the drive.

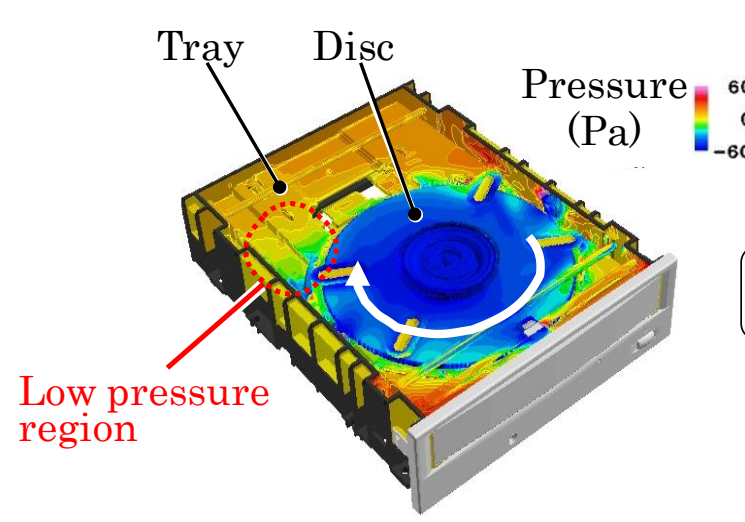

(a) Conventional structure (without tray hole)

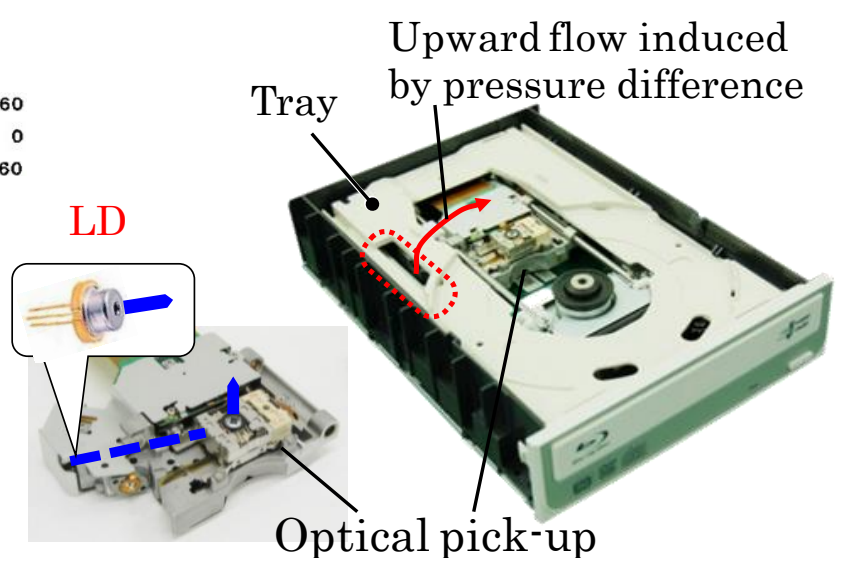

(b) Developed structure

(with additional tray hole)

Figure 4. Comparison of cooling structure for LD

Figure 5 shows the optical disc drive model (slightly simplified geometry model) used in this study, a 3D model of the tray, the medial-surface model generated from the 3D model, and the element model that is generated by dividing the medial-surface into elements. We confirmed that an adequate medial-surface (zero-thickness) and element model are generated, although the 3D model has some holes and steps. We also confirmed in Figure 6 that adequate medial-surface and element models are generated from the product CAD model which has many small holes, ribs, and steps. 


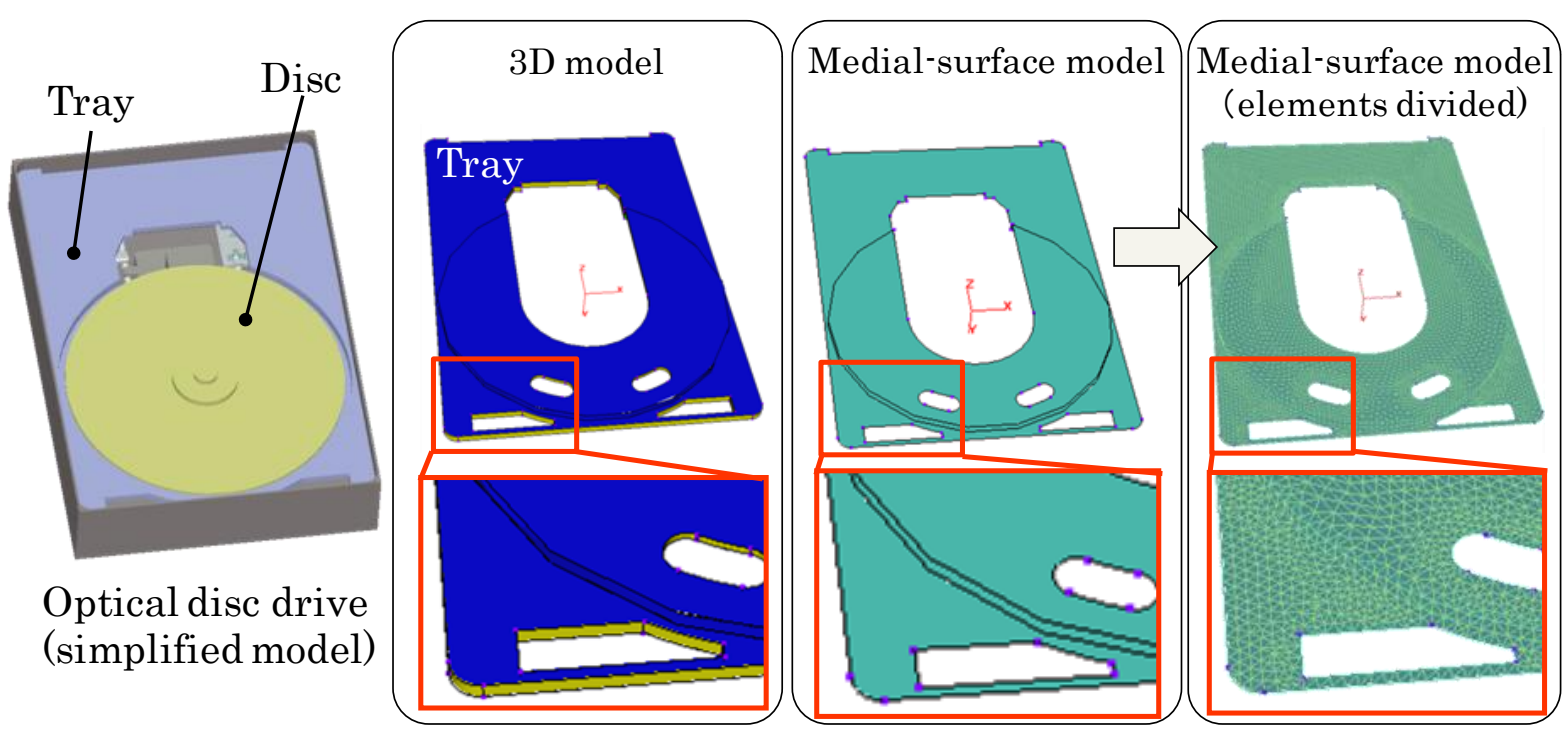

Figure 5. Medial-surface model for optical disc drive (simplified model)
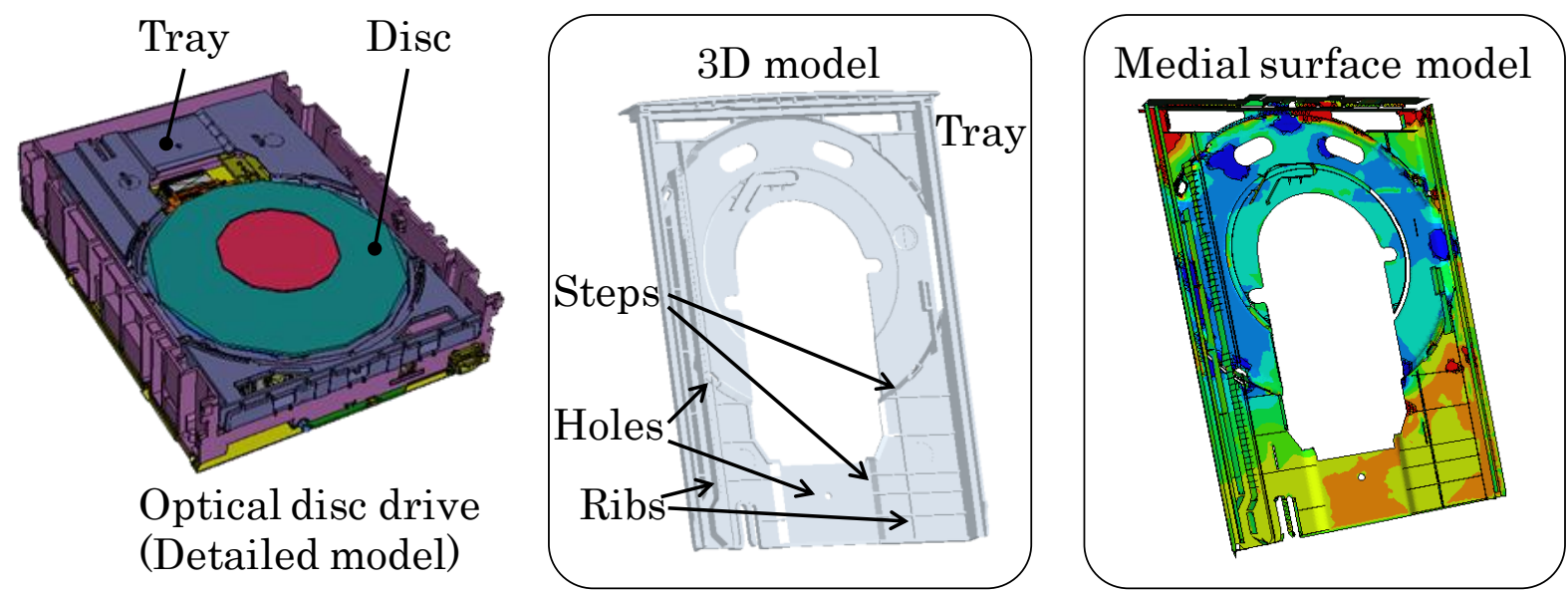

Figure 6. Medial-surface model for optical disc drive (actual product model)

The pressure distribution of the (a) front side, (b) back side, and (c) pressure difference distribution between the front and back side sides are shown on the medial surface in Figure 7. Here, the pressure difference is defined as the "front side value" - "back side value" as the point of view. The pressure data is given by the CFD simulation obtained from using a 10,000 step time-averaging procedure, after the initial 10,000 steps from the quasi-steady state flowfield data. 


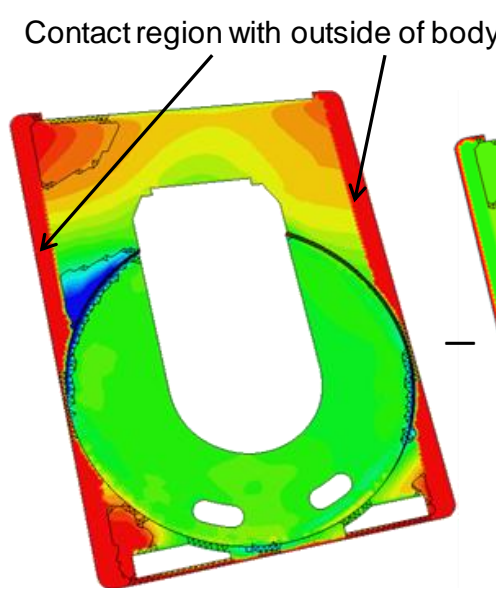

(a) Front side pressure

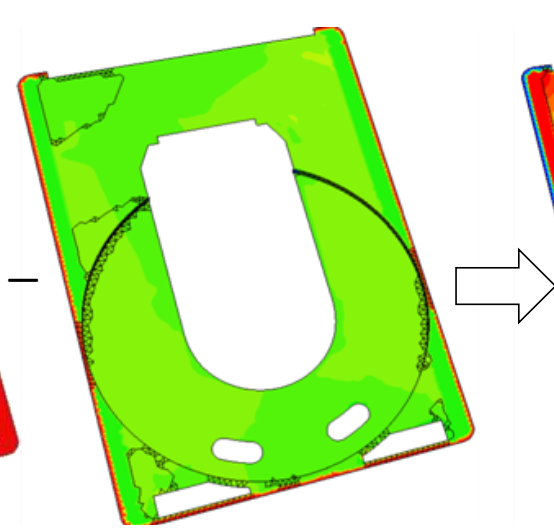

(b) Back side pressure

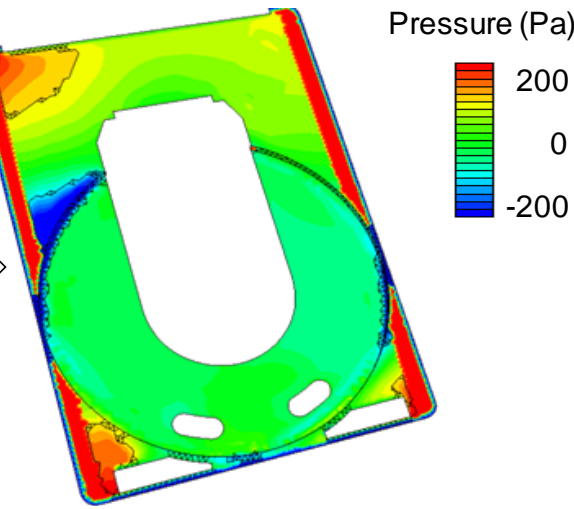

(c) Pressure difference between front and back sides

Figure 7. Pressure and pressure difference distributions on tray (displayed on medial-surface)

Here, the threshold value for the absolute value of the pressure difference is set, and the feature regions where the pressure difference value exceeds the threshold value are extracted in Figure 8. The threshold value is set to every $20 \mathrm{~Pa}$ between 60-140 $\mathrm{Pa}$. In the case of threshold value (a) $60 \mathrm{~Pa}$, most of the tray is extracted as a feature region, and some small regions are formed such as regions 5 and 6. In the case of (b) $80 \mathrm{~Pa}$, region 1 is small and several small regions such as 9 and 10 remain. Further increases in the threshold value to 100, 120, and $140 \mathrm{~Pa}$ narrow the feature regions and diminish the small regions. From the results in Figure 8, the threshold value necessary to extract the feature region is set at $100 \mathrm{~Pa}$, because the number of regions does not change at $100 \mathrm{~Pa}$.

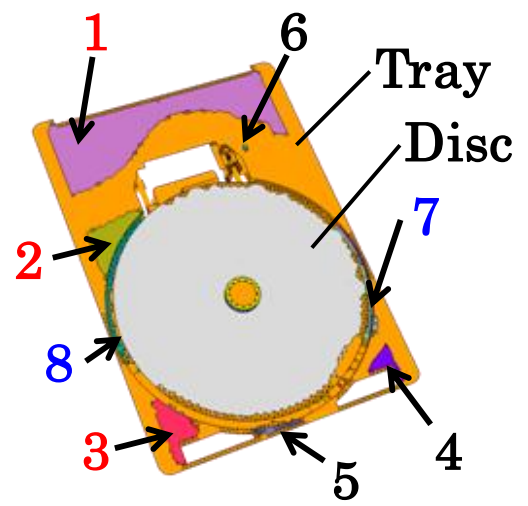

(a)Threshold value $60 \mathrm{~Pa}$

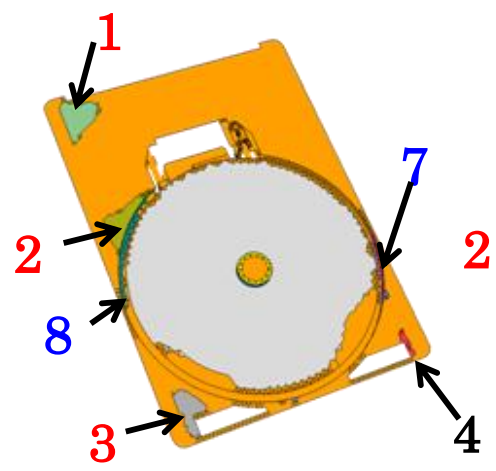

(d) $120 \mathrm{~Pa}$

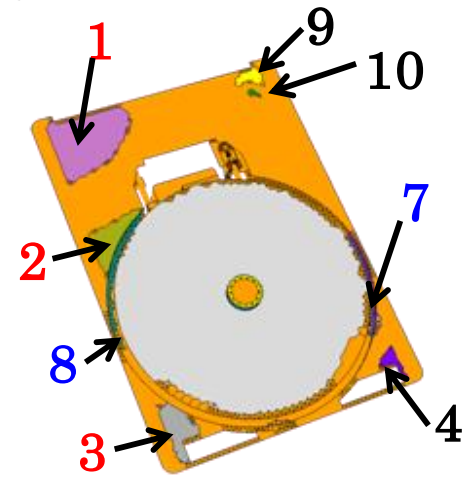

(b) $80 \mathrm{~Pa}$

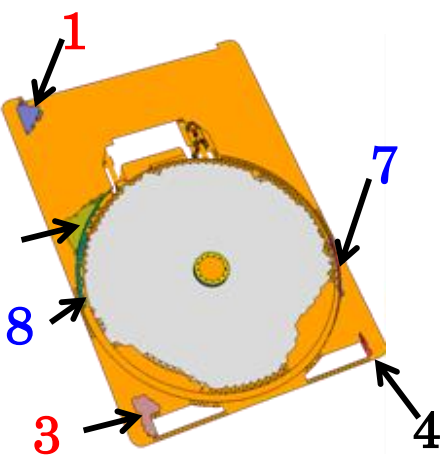

(e) $140 \mathrm{~Pa}$

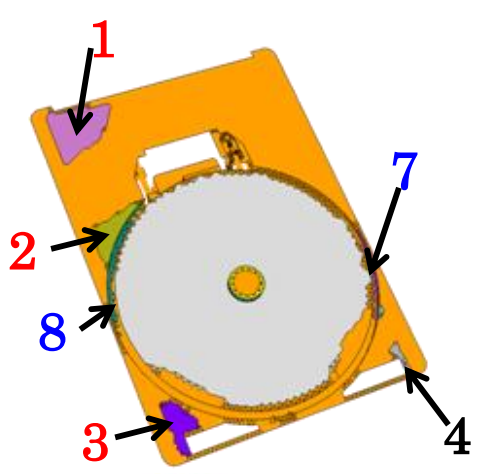

(c) $100 \mathrm{~Pa}$

Figure 8 . Extracted feature regions by setting threshold value for pressure difference value. 
The threshold value of $100 \mathrm{~Pa}$ detects six feature regions (1, 2, 3, 4, 6, and 7). In these six regions, regions 7 and 8 can be neglected, because the design change of the tray structure under the disc is unacceptable in the disc-vibration reduction design, which has been established for many experiments. Region 4 can also be neglected based on its smaller size compared to regions 1,2, and 3. Finally, regions 1, 2, and 3 are selected as the design candidates. Here, region 4 is also evaluated in Table 2 below, but we confirmed that the area integral of pressure difference is smaller relative to the other regions. Thus, neglecting region 4 is acceptable.

The expected contribution to the LD cooling is evaluated by using the two simple-data mining methods mentioned above, (1) distance between LD and additional opening hole candidates and (2) area integral value of pressure difference. Table 1 shows the results based on the distance from the LD to feature regions 1, 2, and 3. Here, the distance between the LD and the feature regions is evaluated by using the distance of each geometrical center position, and the original (no additional opening hole) tray and the LD layout are shown in Figure 9.

Table 1. Evaluation results (1) based on distance between feature regions and laser diode

\begin{tabular}{|c|c|c|}
\hline Rank & Feature region & Distance between feature region and LD (mm) \\
\hline 1 & Region 2 & 25.1 \\
\hline 2 & Region 1 & 51.3 \\
\hline 3 & Region 3 & 107.6 \\
\hline
\end{tabular}

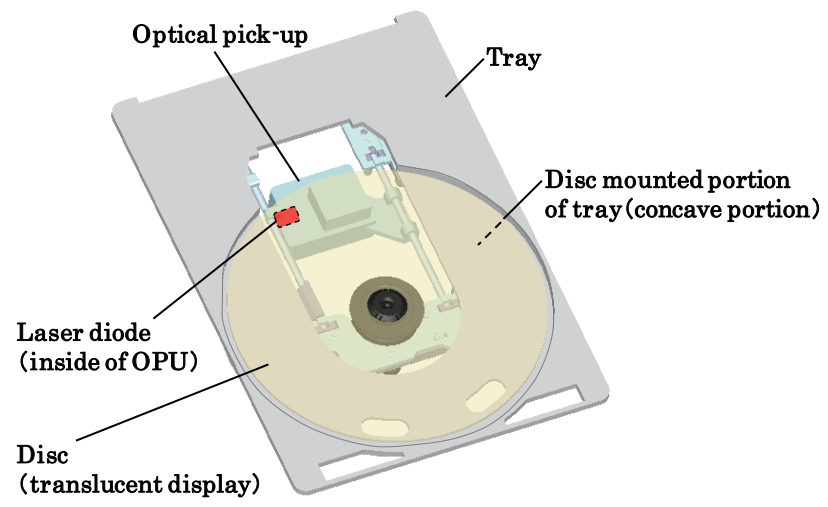

Figure 9. Structure and layout of tray, disc, and laser diode

Table 2 also shows the results based on the area integral value of pressure difference between the front and back sides.

Table 2. Evaluation result (2) based on area integral value of pressure difference

\begin{tabular}{|c|c|c|}
\hline Rank & Feature region & Area integral value of pressure difference $(\mathrm{N})$ \\
\hline 1 & Region 2 & 0.121 \\
\hline 2 & Region 1 & 0.078 \\
\hline 3 & Region 3 & 0.054 \\
\hline$(4)$ & Region 4 & 0.007 \\
\hline
\end{tabular}

From the results in Tables 1 and 2, the distance between the LD and region 2 is small, and the area integral value of pressure difference is also large. In region 2 , then, the flow rate 
induced by the added opening hole is large and the flow passes near the LD. Thus, the highest cooling effect is expected in region 2. For region 3, in which the distance is large and the area integral value of pressure difference is small, the expected cooling effect to the LD is small.

\section{COMPARISON BETWEEN DATA MINING RESULTS AND ACTUAL PRODUCT DESIGN}

Figure 10 shows the effective cooling structures for the product design developed in 2005. Two new structures are adopted for the LD cooling. The first one is the additional hole on the tray in feature region 2, and the second one is an enlarged gap at the end of the tray in feature region 1 . The first structure induces upward secondary flow and enhances the ventilation flow around the LD, as shown in Figure 11, and the second structure supplies the return flow to the LD from the end of the tray as shown in Figure 12(b). These two structures reduce $2 \mathrm{~K}$ of the LD temperature rise.

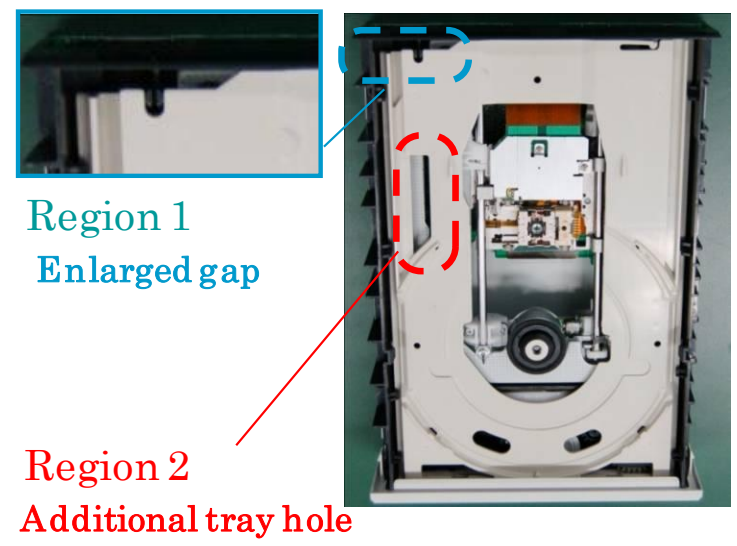

Figure 10. Photograph of actual product

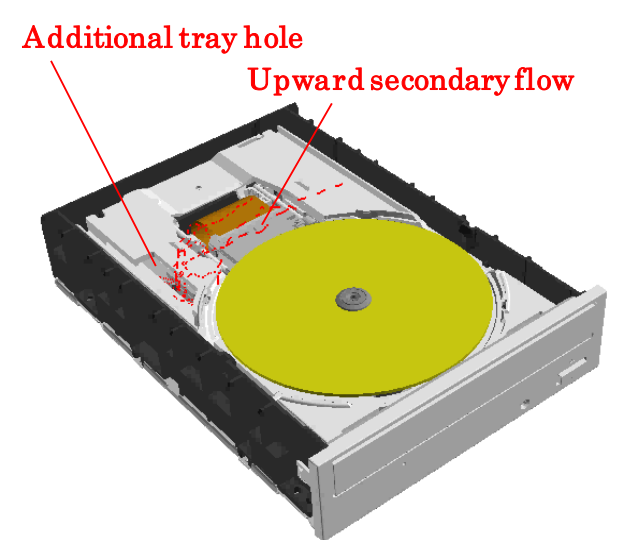

Figure 11. LD cooling contribution by region 2

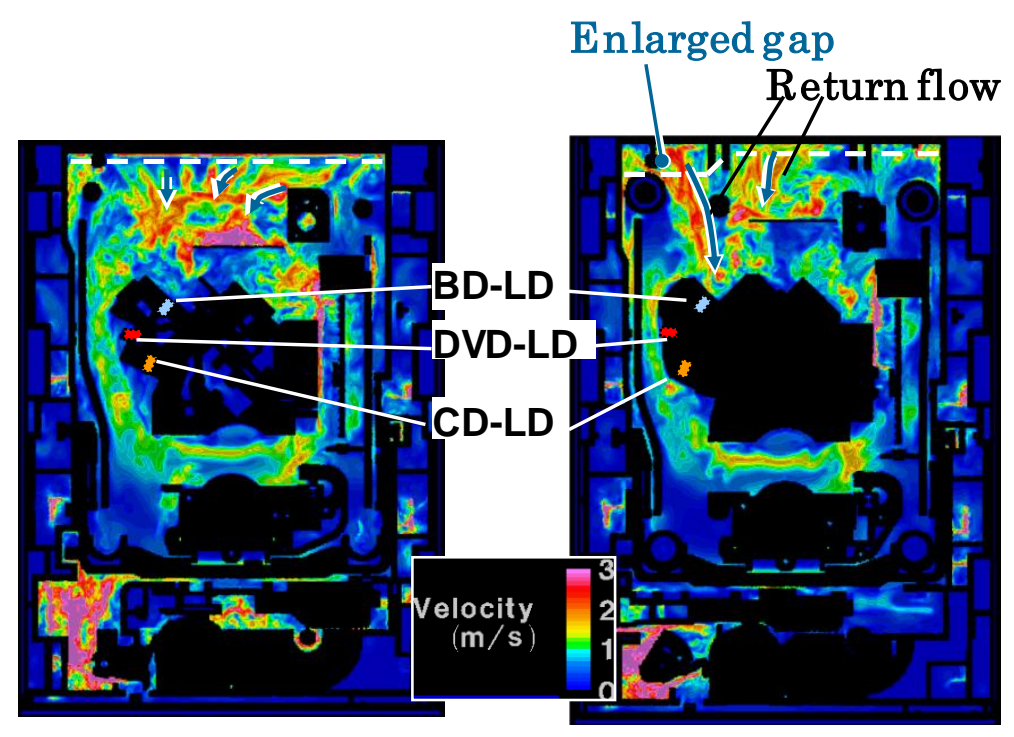

(a) Conventional gap model

(b) Enlarged gap model

Figure 12. LD cooling contribution by region 1 
Then, two of the three structures derived by using this simple data mining method are confirmed to be effective enough for actual product design. The accuracy of effectiveness is 67\%. These structures can not be found before the CFD simulation based on Cartesian grid method has been adopted and the main investigations were done by conducting trial and error experiments. Thus, the method of data mining proposed in this study provides highly accurate proposals of effective designs.

\section{SUMMARY}

A new data mining method for proposing feature regions and design candidates from the CFD simulation results was developed to enhance the contribution of CFD simulations for product development. A design knowledge in which the feature regions are extracted by determining the pressure difference between the front and back sides of specified parts is used in this method. The key technology is the medial-surface method, which is not used for the evaluation of the CFD simulation results. We confirmed that the new method highly accurately predicted the cooling design problem of optical disc drives.

\section{REFERENCES}

[1] N. Isoshima, T. Fujimoto, Y. Abe, M. Watanabe, Y. Yamauchi, and I. Nishida, "Development of cooling structures for Blu-ray Disc compatible optical disc drives using computational fluid dynamics based on Cartesian grid method". ASME-IPACK 2007-33248, 2007.

[2] N. Isoshima, M. Watanabe, M. Ri, Y. Yamada, and K. Sugimoto, "Development of a lownoise and high-performance cooling structure for Full-HD plasma display TV sets". ASME-IPACK 2009-89041, 2009.

[3] W. Nakayama, "Thermal management of electronic equipment: A review of technology and research topics". Appl. Mech. Rev., ASME, 39, 12, 1847-1870, 1986.

[4] S. Ohashi, "Thermal design of flat panel TV (2) (in Japanese) ". Nikkei electronics, 998, 103-108, 2009.

[5] M. Onodera and I. Nishigaki, "A Medial-surface generation technology for CAD-CAE concurrent engineering (in Japanese) ”. Trans. of JSCES, Paper No. 20010037, 2001.

[6] N. Isoshima, T. Fujimoto, Y. Abe, M. Watanabe, and Y. Yamauchi, "Numerical simulation and PIV measurement of the flow-field in optical disc drives". Microsystem Tech.,13, 8-10, 923-931, 2007. 